from Stahl's longer publication, Essential Psychopharmacology (2000), it has the feel of a programmed learning text, a pedagogic style popular in American student texts but relatively unusual in British textbooks of psychiatry. It is beautifully illustrated in colour throughout.

The author states clearly at the outset that his aim is to give the reader a conceptual understanding of the subject. He begins with a gallop through the basics of depression and bipolar disorder, with an emphasis on fundamental neuroscience. This material forms the basis of discussion in the remaining two chapters.

Chapter 2 gives a methodical description of the mechanisms of action of the major classes of antidepressant in current use, augmented by easy-to-follow colour diagrams. The role of the cytochrome P450 enzyme system is clearly and simply illustrated in a review of the pharmacokinetics of antidepressants.

The final chapter, which will probably be the most interesting for advanced readers, deals with the mechanisms of action of newer antidepressants and mood stabilisers. The concept of antidepressants with dual modes of action is illustrated using examples including venlafaxine, mirtazapine and nefazodone. Mention is also made of antidepressants still undergoing development, for example, substance $\mathrm{P}$ antagonists. Stahl concludes with a survey of possible drug-combination strategies for treatment-resistant patients.

This readable and attractive volume should be required reading for all those interested in acquiring a comprehensive understanding of the neuroscience of mood disorders and a working knowledge of the basic pharmacology of antidepressants and mood stabilisers at a neurochemical level. Its clear and simple explanations and illustrations are particularly suited to readers new to the subject, although advanced readers will also find it useful in consolidating their knowledge.

For those with clinical responsibility for prescribing antidepressants and mood stabilisers there is no other book quite like this to stimulate, inform and direct further enquiry. There is a list of further reading, but no source references are provided. Multiple choice questions allow the reader to test retention of the material.

Stahl, S. M. (2000) Essential Psychopharmacology (2nd edn). Cambridge: Cambridge University Press.

M. Bodani Locum Consultant Psychiatrist, St Nicholas Hospital, Collingwood Court, Jubilee Road, Gosforth, Newcastle upon Tyne NE3 3XT, UK

\section{Dangerous Sex Offenders: A Task Force Report of the American Psychiatric Association}

By H. Zonana, G. Abel, J. Bradford, S. K. Hoge, J. Metzner, J. Becker, R. Bonnie \& L. Fitch.

Washington, DC: American Psychiatric Press. 1999. 181 pp. $€ 29.95$ (pb).

ISBN $089042280 \times$

Public opinion on both sides of the Atlantic currently seems to show 'zero tolerance' to sex offenders. Meanwhile, behind both determinate and indeterminate sentencing can lie a pretence that treatment is the purpose of confinement.

The American Psychiatric Association task force has endeavoured to question the 'policing' role given to psychiatrists, while at the same time educating them about sex offenders and their needs. It also examines the effectiveness of classification and treatment and the impact (or lack of impact) of the latter on recidivism.

Many statistics attempting to determine the prevalence of the different paraphilias are provided. As is often the case in such studies, the descriptions of the different diagnoses far outweigh any attempts to understand why such behaviours occur. Treatment is discussed in chapters on cognitive-behavioural and pharmacological method. Psychodynamic treatments are mentioned only in the chapter on recidivism (with its implication that no treatment cures these problems), which contains a brief reference to the lack of psychodynamic research findings. The task force concludes that psychodynamic treatments are not reported because they are not successful.

This seems to me a simplistic review of a complicated field. There is a substantial international psychoanalytic literature that offers much in the way of understanding sexually deviant behaviour, an understanding that has arisen out of careful treatment. Perhaps it is not accredited because it often describes single cases in detail and does not set out to count successes. The failure to acknowledge this literature is particularly apparent in the same chapter on recidivism, in the section on theoretical explanations. It makes no sense to me for the task force to state that there have been very few recent attempts to conceptualise the theoretical causes of sexual offending. Again, the developmental aspects so important to psychoanalysis are completely missed out. The psychological entity is overlooked in the tendency to create ever-growing descriptive lists.

The final two chapters offer some useful questions and information to encourage the reader to think about the issues involved. For example, various scenarios involving a fictitious John Doe examine what to do when he is accused of abusing his 5-year-old daughter, a 13-year-old boy and a 23-year-old woman. The statement in the section on policy recommendations that "sexual predator commitment laws represent a serious assault on the integrity of psychiatry" is certainly thought-provoking.

With reservations, the report serves as a review of the current struggle with a very real and complex problem for which there is no simple answer.

Anne Zachary Consultant Psychiatrist in Psychotherapy, The Portman Clinic, 8 Fitzjohn's Avenue, London NW3 5NA, UK

\section{Male Victims of Sexual Assault (2nd edn)}

Edited by Gillian C. Mezey \& Michael B. King. Oxford: Oxford Medical Publications. 2000. I6I pp. $€ 37.50$ (hb). ISBN 0192629328

"Male rape is a taboo subject" is the first sentence of the first edition of this volume, published in 1992. It is partly a tribute to the editors that times have changed, with the subject now being more openly

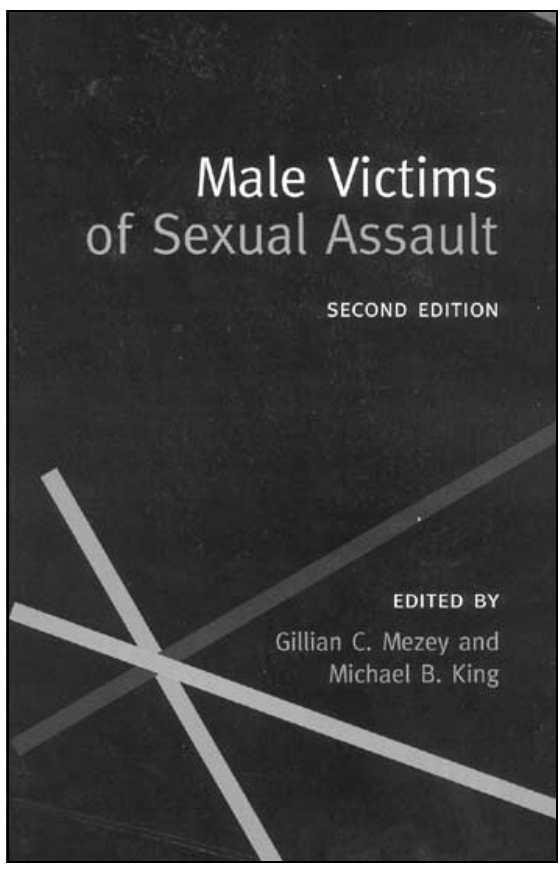

